\section{Sino-Swiss Workshop on Bioinformatics}

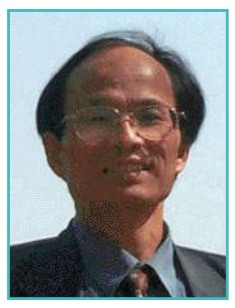

\section{Jingchu Luo}

Center for Bioinformatics, Peking University, Beijing, China

Received 18 March 2013; Published 19 August 2013

As a member of EMBnet, the Global Bioinformatics Network', the Center for Bioinformatics ${ }^{2}$ (CBI) at Peking University has been working very closely with other member organisations for some 15 years. The collaborations with colleagues from the Swiss Institute of Bioinformatics (SIB) ${ }^{3}$ in activities such as exchange visits and Sino-Swiss workshops are among the most memorable and productive.

The first Sino-Swiss Workshop on Bioinformatics (SSWB) was held at CBI in November 1999. Members of the Swiss delegation included Amos Bairoch, the creator of the Swiss-Prot database, and Victor Jongeneel, the then Node manager of EMBnet Switzerland. The late Professor Xiaocheng $\mathrm{Gu}$, founder of the $\mathrm{CBI}$ and pioneer of Chinese bioinformatics, hosted the workshop.

A year later, a follow-up SSWB was held in Switzerland, jointly funded by the National Natural Science Foundation of China $^{4}$ (NSFC) and the Swiss National Science Foundation ${ }^{5}$ (SNSF). The delegation of eight Chinese scientists visited SIB's different partners in Geneva, Lausanne, Basel and Zurich.

Amos Bairoch hosted my presentation in Geneva. His story in running SwissProt, and the high curation standard he brought to the biological database community, are legendary. "It is like a dream!", I started, "I met Amos the first time in London, at the Imperial Cancer Research Fund. We met again last year in Beijing at the first SSWB."

\footnotetext{
1 http://www.embnet.org/

2 http://www.cbi.pku.edu.cn/

3 http://www.isb-sib.ch/

4 http://www.nsfc.gov.cn/

5 http://www.snf.ch/
}

As I went on telling the story, "I saw with the amazement that Amos took out a laptop from his rucksack, typed in the plant peptide sequences he'd spotted from one of our posters, and ran a BLAST search against Swiss-Prot, which was installed on his laptop". I was witnessing the creation of the master copy of Swiss-Prot - as the legend has it, the database was all on his laptop. I was left wondering, if there were a match in the BLAST output, what on the poster could be a new piece of annotation to be added to the sequence! The next stop of our visit was Lausanne, where the 2000 EMBnet Annual General Meeting (AGM) was held. The visit was hosted by Ron Appel, the executive director of SIB, and Victor Jongeneel, on behalf of EMBnet Switzerland. Boqin Qiang, a senior geneticist from the Chinese Academy of Medical Sciences $^{6}$ (CAMS), gave an introduction about China's involvement in the on-going international Human Genome Project. We then visited the bioinformatics group at Roche in Basel, hosted by Martin Ebeling, the former Node manager of EMBnet Germany.

It was a fruitful visit. With the help of Amos and his colleagues at SIB, we set up an ExPASy mirror at $\mathrm{CB}$ in 2000, making the resource much easier to access for users in China and across Asia.

The collaborations between both sides have continued since then, and I met Amos again several times at various meetings. The photo with Amos and Terri Attwood (Figure 1) was taken at the 2007 EMBnet AGM in Malaga, a southern city in Spain. We sent two young Chinese bioinformaticians to SIB for their post-doctorate training in Lausanne; they came back and developed their

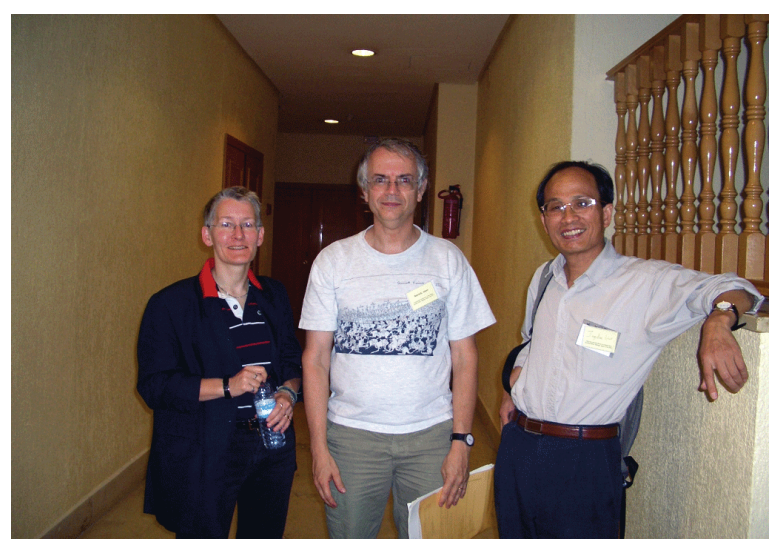

Figure 1. A photo taken during the 2007 EMBnet AGM, in Malaga, Spain. From left to right: Teresa Attwood, Amos Bairoch, Jingchu Luo

6 http://www.cams.ac.cn 
own labs. Laurent Falquet, the next Node manager of EMBnet Switzerland, visited Beijing in 2008, and gave our students a well-received course.

Soon after the New Year of 2013, a Swiss delegation of ten bioinformaticians, led by Ron Appel, visited Shanghai. Amos joined the delegation as the director of Computer Analysis and Laboratory Investigation of Protein of Human Origin', now working on a new protein database NeXtProt' The delegation was also joined by loannis Xenarios, who manages SwissProt and ExPASy,

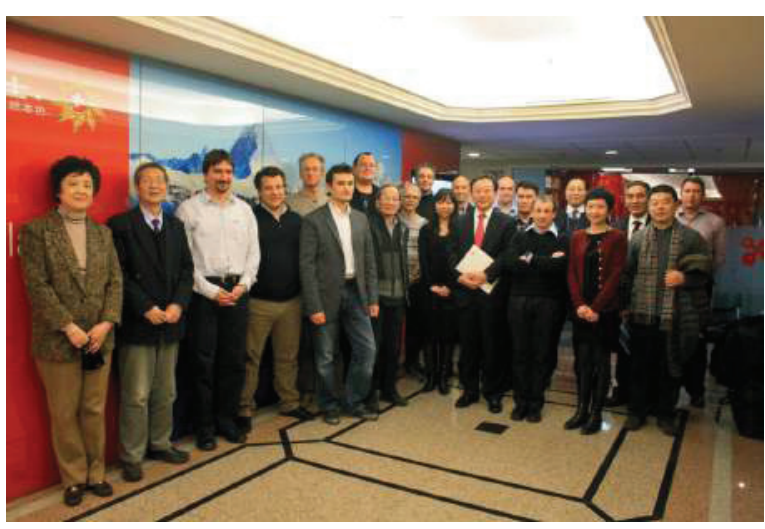

Figure 2. Participants of the Sino-Swiss workshop on Bioinformatics held on 8 January 2013, at Swissnex China in Shanghai.

as well as SIB's HPC infrastructure, VITAL-IT'. It was a short visit of only three days, yet a successful workshop was held in the small town of Taicang, north of Shanghai, organised by Swissnex China, Switzerland's outpost for Science, Technology and Culture in China, and the Taicang Institute of Life Sciences Information ${ }^{10}$ (TILSI), a new bioinformatics service centre set up by Weimin Zhu, the former head of Database Applications at the EBI. The Chinese delegation was led by the President of CMAS, Xuetao Cao, joined by ten bioinformaticians from organisations at the Chinese Academy of Sciences ${ }^{11}$ (CAS), CBI, TILSI and CMAS.

Although time was limited for the talks, participants from both sides did learn a great deal about each others' work in research, development, service and education in bioinformatics, genomics, proteomics, molecular modelling and phylogenetics. Representing the national Node of EMBnet China, my presentation focused on the bioinformatics service and education in which we have been involved during the past 15 years (Figure 3).

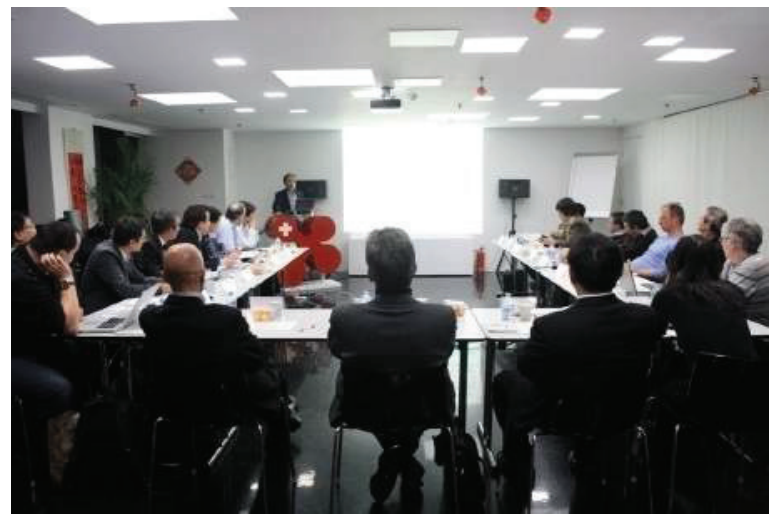

Figure 3. Presentation by Jingchu Luo at the Sino-Swiss Workshop, 2013

It was proposed as early as in 1999 by a Chinese Academician, Bailin Hao, to build a National Bioinformatics Centre of China (NBCC), which would play the same role as the NCBI and $\mathrm{EBI}$, providing bioinformatics resources, services and training. Although many efforts have been made toward this goal, its organisational model and infrastructure have yet to be determined and built. The operational model of the proposed NBCC is one of the key questions. At this workshop, Chinese delegates listened with great interest to the description of the federated model of SIB. A task force, funded by CAS, was recently created, with the goal of conducting a thorough survey to address some of the important questions, and produce recommendations to funding agencies. The task force is in the process of organising a trip to SIB in 2013, to learn more from Swiss colleagues about building and running a successful bioinformatics centre at national level. Trips to NCBI and EBI are also planned.

\section{Acknowledgements}

Thanks to WM Zhu and TK Attwood for critical reading of the manuscript.

\footnotetext{
7 http://www.isbsib.ch/groups/geneva/calipho-bairoch. html

8 http://www.nextprot.org/

9 http://www.vital-it.ch

10 http://www.tilsi.org/

11 http://www.cas.cn/
} 